\title{
BMJ Open Effectiveness and reporting standards of psychological interventions for improving short-term and long-term pain outcomes after total knee replacement: a systematic review
}

Katie Whale, ${ }^{1,2}$ Vikki Wylde, ${ }^{1,2}$ Andrew Beswick, ${ }^{2}$ James Rathbone, ${ }^{3,4}$ Kavita Vedhara, ${ }^{3}$ Rachael Gooberman-Hill ${ }^{1,2}$

To cite: Whale $\mathrm{K}$, Wylde $\mathrm{V}$, Beswick A, et al. Effectiveness and reporting standards of psychological interventions for improving short-term and long-term pain outcomes after total knee replacement: a systematic review. BMJ Open 2019;9:e29742. doi:10.1136/ bmjopen-2019-029742

- Prepublication history and additional material for this paper are available online. To view these files, please visit the journal online (http://dx.doi org/10.1136/bmjopen-2019029742).

Received 13 February 2019 Revised 01 July 2019 Accepted 18 October 2019

Check for updates

(C) Author(s) (or their employer(s)) 2019. Re-use permitted under CC BY-NC. No commercial re-use. See rights and permissions. Published by BMJ.

For numbered affiliations see end of article.

Correspondence to

Dr Katie Whale;

katie.whale@bristol.ac.uk

\section{ABSTRACT}

Objectives To assess the effectiveness and reporting standards of psychological interventions for improving outcomes after total knee replacement (TKR).

Design Medline, Embase, and PsycINF0 were searched from inception to up to 9 May 2019 with no language restrictions applied. Randomised controlled trials (RCTs) assessing the effectiveness of psychological interventions for short-term and long-term postoperative pain after TKR were included. Screening, data extraction, and assessment of methodological quality were performed in duplicate by two reviewers. The primary effectiveness outcome was postoperative pain severity and the primary harm outcome was serious adverse events. Secondary outcomes included function, quality of life, and psychological well-being. Reporting standards were assessed using the Template for Intervention Description and Replication (TIDieR) checklist for intervention reporting.

Results 12 RCTs were included, with a total of 1299 participants. Psychological interventions comprised music therapy (five studies), guided imagery and music (one study), hypnosis (one study), progressive muscle relaxation with biofeedback (one study), pain coping skills programme (one study), cognitive-behavioural therapy (two studies), and a postoperative management programme (one study). Due to the high heterogeneity of interventions and poor reporting of harms data, it was not possible to make any definitive statements about the overall effectiveness or safety of psychology interventions for pain outcomes after TKR. Conclusion Further evidence about the effectiveness of psychological interventions for improving pain outcomes after TKR is needed. The reporting of harm outcomes and intervention fidelity is currently poor and could be improved. Future work exploring the impact of intervention timing on effectiveness and whether different psychological approaches are needed to address acute postoperative pain and chronic postoperative pain would be of benefit.

PROSPERO registration number CRD42018095100.

\section{INTRODUCTION}

Total knee replacement (TKR) is the second most commonly performed elective
Strengths and limitations of this study

- Inclusion of randomised controlled trials to evaluate all available evidence and the identification of published protocols to highlight ongoing research likely to add to the evidence base.

- Evaluation of intervention reporting standards identified areas for improvement for future studies.

- Limited opportunities for pooling of data in meta-analysis due to heterogeneity of included interventions.

procedure in the UK with nearly 100000 procedures performed in annually. ${ }^{2}$ TKR is performed to reduce pain and improve functional ability, predominately for people with osteoarthritis. TKR is a successful operation for many patients, with patient satisfaction ranging between $81 \%$ and $89 \% .^{3-5}$ However, acute postoperative pain after TKR is common, with over half of patients reporting moderate-severe pain in the first 3 days post operation. ${ }^{6}$ In the longer term, previous studies have demonstrated that up to $20 \%$ of patients experience unfavourable pain outcomes between 3 months and 5 years postoperatively. $^{7-9}$ Chronic pain has been shown to be the strongest predictor of dissatisfaction with TKR. ${ }^{7}$ Pain after TKR is linked to decreased activity levels, which negatively impacts recovery, and can have a substantial adverse impact on quality of life and wellbeing. ${ }^{10}$ In addition, treatment and investigations in relation to chronic pain come at cost to the National Health Service (NHS). ${ }^{11}$ Between 2003 and 2017, the National Joint Registry recorded 28717 first revisions after primary TKR, ${ }^{1}$ often with little benefit for relief of pain. ${ }^{12}$ The reduction and treatment of postsurgical pain after TKR is therefore a 
key focus of research to optimise outcomes and improve patient satisfaction.

Chronic pain after TKR is multifactorial in aetiology, with causes including mechanical, biological, surgical, and psychological factors. ${ }^{13-16}$ In the field of chronic pain management, multidisciplinary approaches including multimodal combinations of analgesics, physical therapy, behavioural therapy, and psychological therapy have been shown to be superior to unimodal approaches such as analgesics only. ${ }^{17-19}$ Conventionally, management of pain after surgery has focused on mechanical and biological aspects through the use of analgesic interventions and physiotherapy. ${ }^{21}{ }^{21}$ However, there is increasing awareness of the potential for psychological interventions to be implemented alongside surgery in the preoperative, perioperative, or postoperative period to improve postsurgical outcomes. Psychological interventions may be of particular benefit to patients receiving TKR due to the role that psychological risk factors play in surgical outcomes. Previously conducted systematic reviews and prospective cohort studies indicate that increased anxiety, depression, pain catastrophising (magnification of the pain experience, rumination on the pain, feelings of helplessness), and a lack of active coping strategies as risk factors for increased postoperative pain after TKR beyond the acute recovery period. ${ }^{22-25}$

A previous meta-analysis and systematic review of psychological interventions alongside surgery, including orthopaedic procedures, found that relaxation and guided imagery therapy were effective in improving physical and psychological outcomes, including reduced acute postoperative pain levels and analgesic use. ${ }^{26}$ However, this previous review included a range of surgical procedures when looking at the effectiveness of psychological interventions, including abdominal, cardiac, and lumbar and spinal surgery. This makes it challenging to draw specific conclusions about the utility of psychological interventions for patients receiving TKR. A systematic review of randomised controlled trials (RCTs) published in June 2018 with no published protocol found mixed evidence for the effectiveness of psychological interventions for improving outcomes after TKR and total hip replacement (THR) ${ }^{27}$ However, this review evaluated TKR and THR together with one included study including TKR patients only, and therefore the findings are limited as these are two different surgical procedures with distinct indications and outcomes. ${ }^{28}$ To date, no systematic review has been conducted to evaluate the effectiveness of psychological interventions for patients undergoing primary TKR. Psychological interventions targeting pain may be of particular benefit to TKR patients due to the high incidence of chronic pain after surgery.

Potential challenges in evaluating the literature on psychological interventions are a lack of robust intervention reporting and heterogeneity in the use of psychological terminology. A previous analysis of RCTs found that only $29 \%$ of non-pharmacological interventions provided adequate completeness of intervention description. $^{29}$ Without thorough reporting, other researchers are unable to replicate or build on research findings, and synthesis of findings in systematic reviews and meta-analysis is difficult. Psychological interventions are complex and often involve varying intensity, doses, duration, and mode of delivery. Due to this complexity, it is important that published studies provide clear descriptions of the content of the interventions to ensure that interventions can be replicated and results of any evaluations are transparent and open to full interpretation. To address consistency and transparency in reporting of interventions, a checklist and guidance entitled Template for Intervention Description and Replication (TIDieR) has been developed. ${ }^{30}$ TIDieR was designed for all types of intervention in health; it provides the minimum recommended items for describing an intervention to ensure replicability and can be used in reporting of interventions and in assessment of reporting quality. Using TIDieR to assess the reporting of psychological interventions provides a structured, objective assessment of current reporting standards and may help to identify areas for improvement.

The primary aim of this systematic review was to assess the clinical effectiveness of psychological interventions for improving pain outcomes after TKR. The secondary aim was to evaluate the reporting quality of these interventions assessed using the TIDieR checklist.

\section{METHODS}

Conduct of the systematic review followed guidance from the Cochrane handbook ${ }^{31}$ and reporting was in accordance with the Preferred Reporting Items for Systematic Reviews and Meta-Analyses ${ }^{32}$ (PRISMA). The PRISMA checklist can be found in online supplementary appendix 1.

\section{Searches}

Systematic literature searches were conducted using the Ovid Gateway to access Medline, Embase and PsycINFO. Searches were conducted from inception to 9 May 2019, and no language restrictions were applied. Search terms used are provided in online supplementary appendix 2. Institute of Scientific Information (ISI) Web of Science was used to check citations of key reviews and studies. Excluded studies included those reported only as dissertations or conference abstracts. Articles that were unobtainable and study protocols were also excluded.

\section{Eligibility criteria}

The following criteria were applied to determine eligibility of studies for inclusion in the review:

- Population: adults undergoing primary TKR.

- Intervention: any psychological intervention delivered preoperatively, perioperatively, or postoperatively to patients. Psychological interventions were defined as six categories: behavioural, cognitive, relaxation/ mindfulness, group-based psychological support, 
social skills training, and psychotherapy/counselling. Multimodal and complex interventions with psychological components were also considered eligible.

- Control: active treatment or control treatment (eg, standard care, placebo, no treatment).

- Outcomes: assessment of postoperative pain severity (no time limit placed on assessment duration/ follow-up).

- Study type: RCT.

\section{Psychological interventions}

Psychological interventions are defined as using specific principles and techniques hypothesised to improve psychological well-being or a reduction in symptoms associated with psychological difficulties, ${ }^{33}$ such as pain. Interventions eligible for inclusion included, but were not restricted to, cognitive-behavioural therapies; behavioural interventions; acceptance-commitment therapy; social skills training; relaxation therapies; mindfulness; psychodynamic; counselling; and interpersonal therapies. Excluded therapies included, but were not restricted to, didactic education or education designed to impart knowledge; pharmacological therapy; physiotherapy; spiritual healing (eg, reiki); complementary and alternative medicine.

\section{Screening}

All records identified through the searches were imported into Endnote X8 (Thomson Reuters) and duplicates removed. All articles were screened initially by one researcher (KW or VW), and articles that were identified as clearly not relevant were excluded. Potentially eligible articles were screened at abstract and full-text level by two reviewers independently (KW and VW). Screening results were then compared with any discrepancies discussed between the reviewers. If consensus could not be achieved, then a third independent reviewer was consulted (KV). Reasons for exclusion were recorded in Microsoft Excel.

\section{Data extraction}

Relevant data were extracted onto a standardised proforma by a researcher (KW, VW, or JR). Completed data extraction forms were then checked against the source article by a second reviewer (KW, VW, or JR). Extracted data included: study design, country, date, study population, content of the intervention, primary and secondary outcome data, measures used and data collection timepoints, information for assessment of risk of bias, and reporting standards assessed by the TIDieR checklist. If a study included TKR patients but did not provide disaggregated data, then a single email was sent to the corresponding author to enquire if these data were available. If no response was received or the data were not available, then the study was excluded.

\section{Outcomes}

Following Cochrane guidance, ${ }^{31}$ this review used one primary outcome for effectiveness and one for harm. The primary effectiveness outcome was knee pain severity, measured at any time-point after surgery. No limits were placed on the measures used to assess this outcome or on the follow-up duration period. The primary harm outcome was the occurrence of serious adverse events. Our definition of a serious adverse events was any untoward medical or psychological occurrence that met any of the following conditions:

- Resulted in death.

- Was life-threatening.

- Required inpatient hospitalisation or prolongation of existing hospitalisation.

- Resulted in persistent or significant disability/ incapacity.

- Resulted in heightened levels of psychological distress from participants in the intervention.

Secondary outcomes included health-related quality of life, psychological well-being/status, and reporting standards. Reporting standards for interventions were assessed using the TIDieR guidelines and checklist. ${ }^{30}$ TIDieR provides a template of minimum reporting standards for intervention description and replication. The 12-item checklist is applied on a presence/absence basis with each item recorded as yes, no, or partial. The guide provides additional detail on elaboration for each item, and examples of good reporting.

\section{Risk of bias and reporting standards}

Risk of bias for RCTs was assessed using the Cochrane risk of bias tool, which assesses risk of bias across six domains: selection, performance, detection, attrition, reporting, and other. ${ }^{34}$

\section{Strategy for data synthesis}

At the protocol stage, meta-analysis was planned if an appropriate number of studies were identified with similar intervention and comparator groups, and comparable outcome data. If pooling of outcome data was not appropriate, a narrative synthesis was planned. Full details of the planned analysis strategy are provided in the PROSPERO record.

At analysis stage, opportunities for meta-analysis were limited by the heterogeneity in the content, duration and intensity of the interventions. Therefore, a narrative synthesis was conducted.

\section{Patient and public involvement (PPI)}

This research was conducted in a musculoskeletal research unit within which research priorities and delivery are identified and developed with ongoing PPI. This involvement takes place through the activities of the Patient and Public Partnership in Research who have identified outcomes after knee replacement to be a key research area that they wish to see explored. Once the findings of this review have been published, the research team will work in collaboration with the patient involvement group to design dissemination approaches so that findings reach a wide audience. 


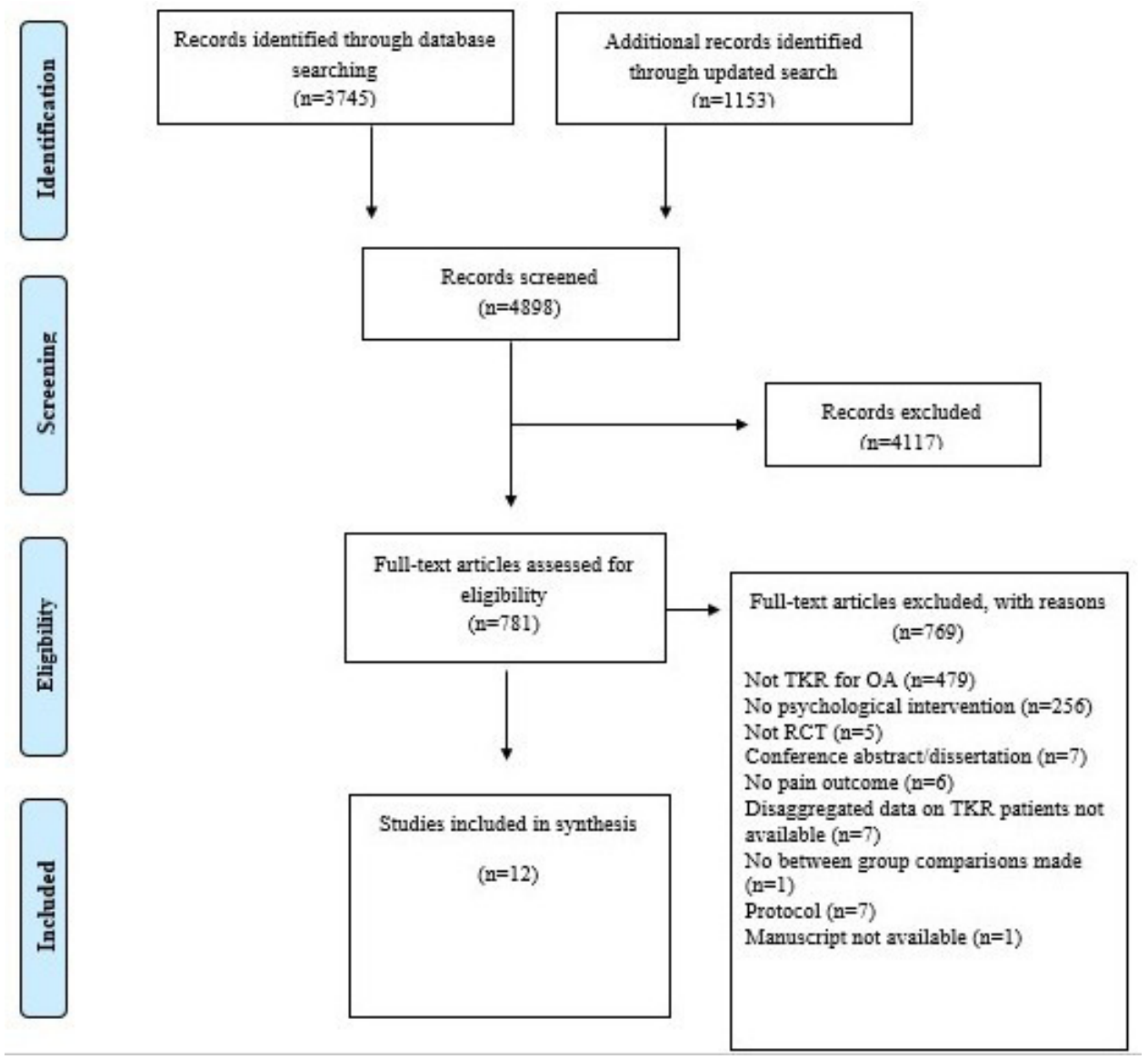

Figure 1 Preferred Reporting Items for Systematic Reviews andMeta-Analyses flow chart. OA, osteoarthritis; RCT, randomised controlled trial; TKR, total knee replacement.

\section{RESULTS}

Searches identified 4898 articles, and 781 full-text articles were assessed for eligibility. Twelve RCTs with a total of 1299 participants were eligible for inclusion. ${ }^{35-46} \mathrm{~A}$ PRISMA flow diagram is provided in figure 1.

\section{Study characteristics}

An overview of study characteristics is provided in table 1 . Included studies were from the USA $(n=6)$, Taiwan $(n=2)$, the UK $(n=2)$, China $(n=1)$, and Malaysia $(n=1)$. The number of centres was reported for 11 studies: 9 studies were conducted in a single centre, 1 study was conducted in 2 centres, and 1 study was conducted in 5 centres. Sample sizes for the included studies ranged from 24 to 402 participants, with a median of 71 . One study included interventions delivered perioperatively, six postoperatively, and five preoperatively and postoperatively. Four studies conducted follow-up assessments during inpatient stay only (maximum 72 hours), one study 5 days post operation, five studies 6 months post operation, and one study 12 months post operation. One study collected outcome measures at the time of intervention only (postoperative physiotherapy) but did not state the timing post operation. The most commonly used pain outcome measure was the Pain Visual Analogue Scale (VAS)/Numerical Rating Scale (NRS) ( $n=9)$; other measures used were the Pain VAS $(n=4)$, the Western Ontario and McMaster
Universities Osteoarthrtisi Index (WOMAC) Pain Scale $(n=4)$ and the short-form McGill pain questionnaire $(\mathrm{n}=1)$. An overview of study findings is provided in online supplementary appendix 3 . The primary harm outcome of serious adverse events was reported in one study only but was not defined. Reporting of secondary outcomes was variable. Two studies reported on all secondary outcomes (function, health-related quality of life, and psychological well-being). The most commonly reported secondary outcome reported was knee function, included in five studies. Full details on secondary outcome reporting can be seen in online supplementary appendix 4 .

\section{Methodological quality}

Risk-of-bias assessments for individual studies are shown in figure 2.

\section{Interventions}

Eight studies were classified as relaxation/mindfulness. These studies included music therapy $(n=5)$, hypnosis $(\mathrm{n}=1)$, and progressive muscle relaxation with biofeedback $(n=1)$. One study was multimodal and included guided imagery and music $(\mathrm{n}=1)$. Three studies were classified as cognitive and behavioural and included CBTbased programmes $(n=2)$ and a postoperative management programme comprising motivational interviewing to improve self-efficacy and goal attainment $(n=1)$. One 


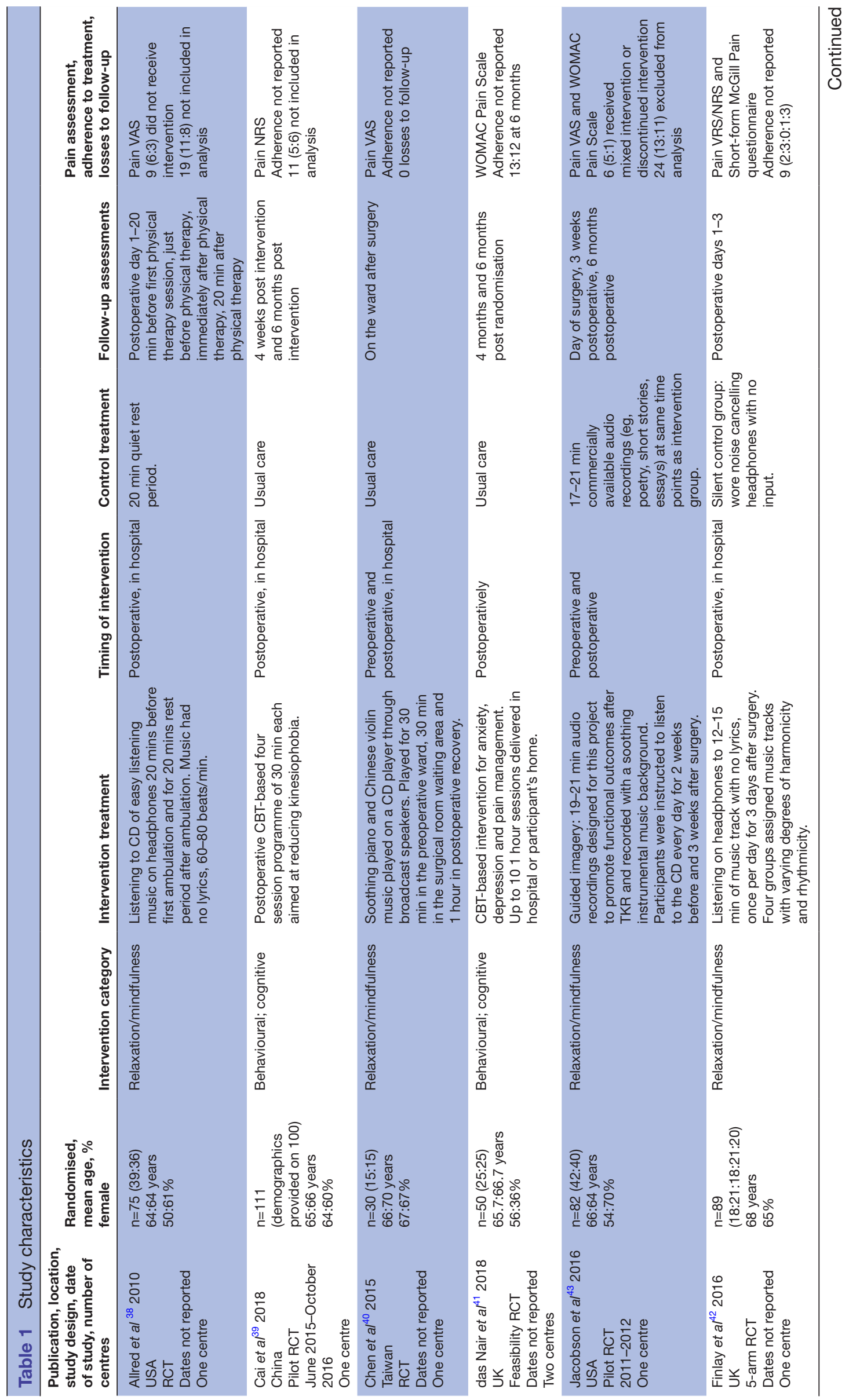




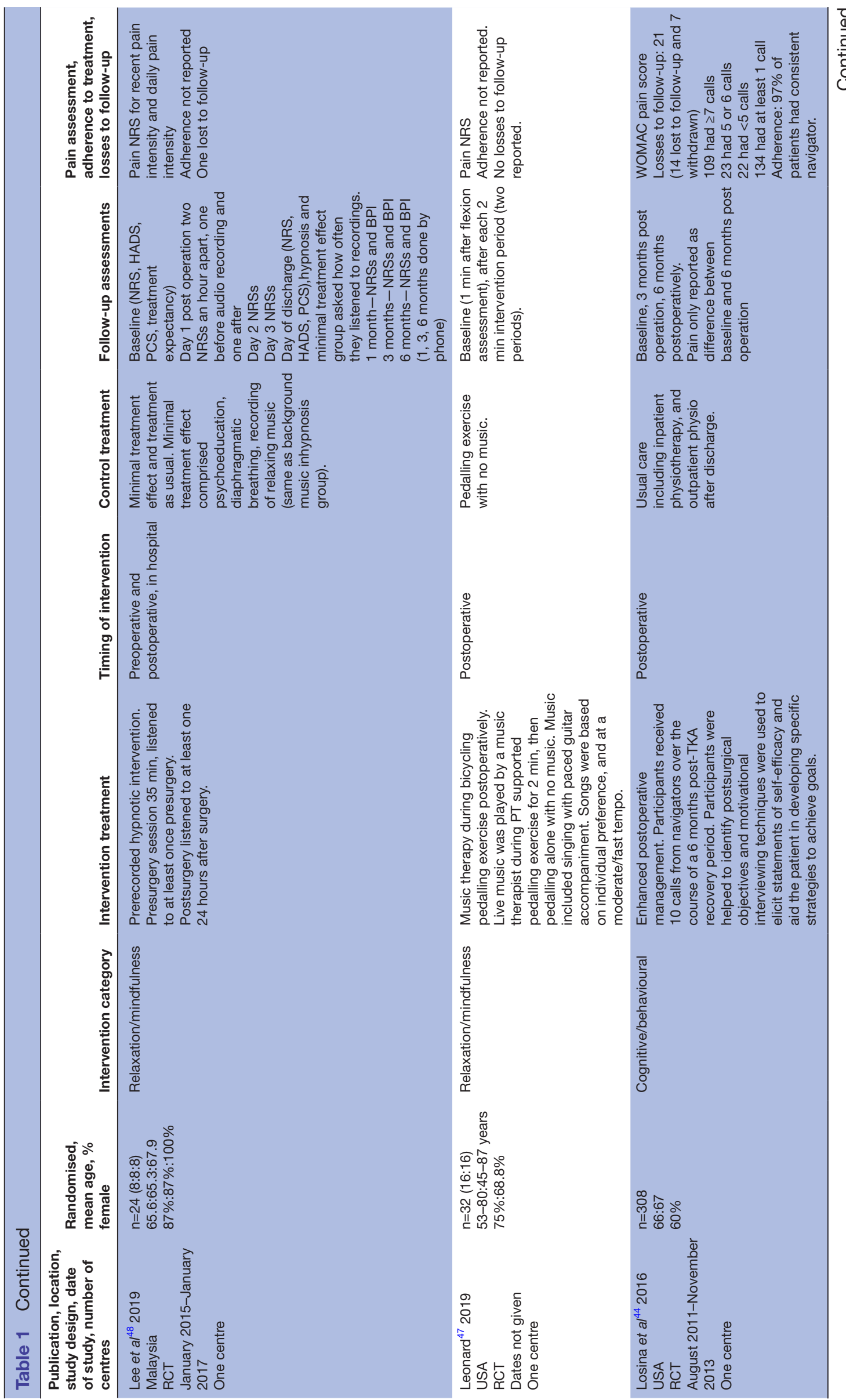




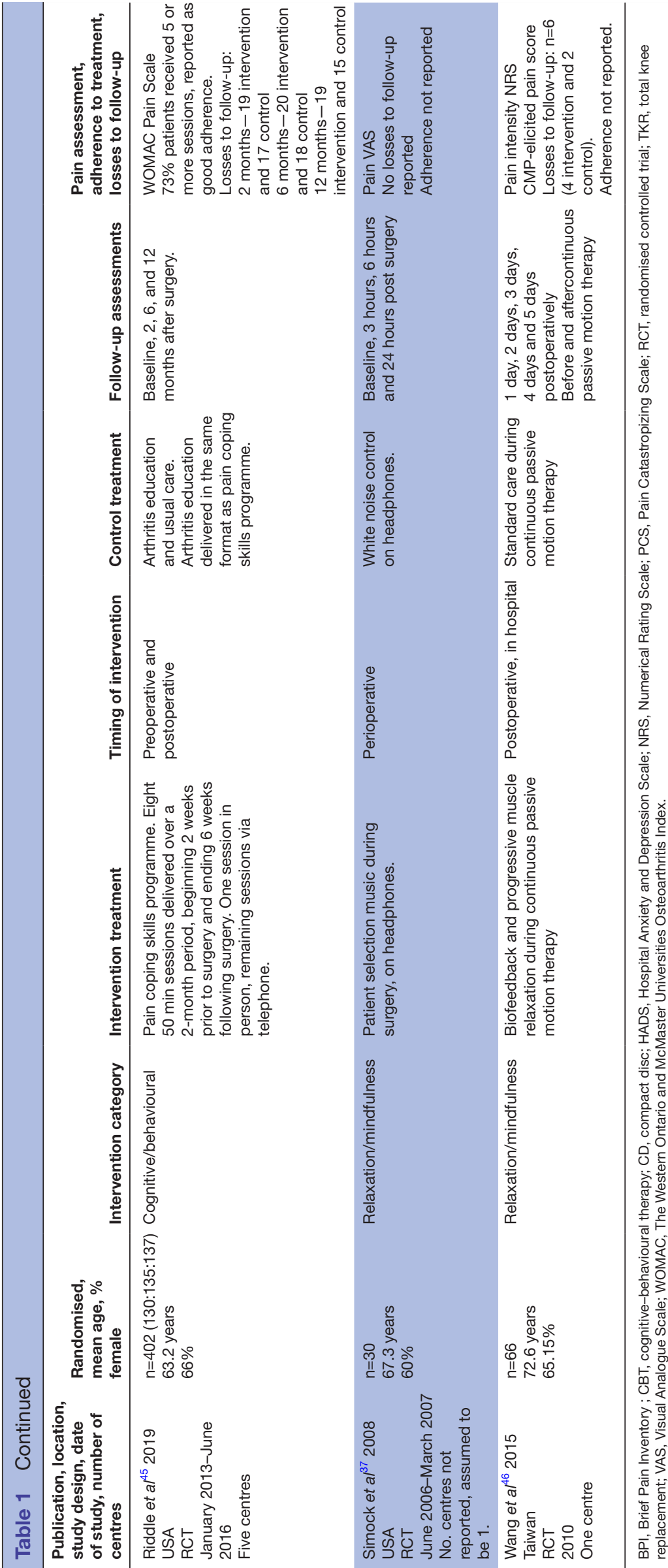




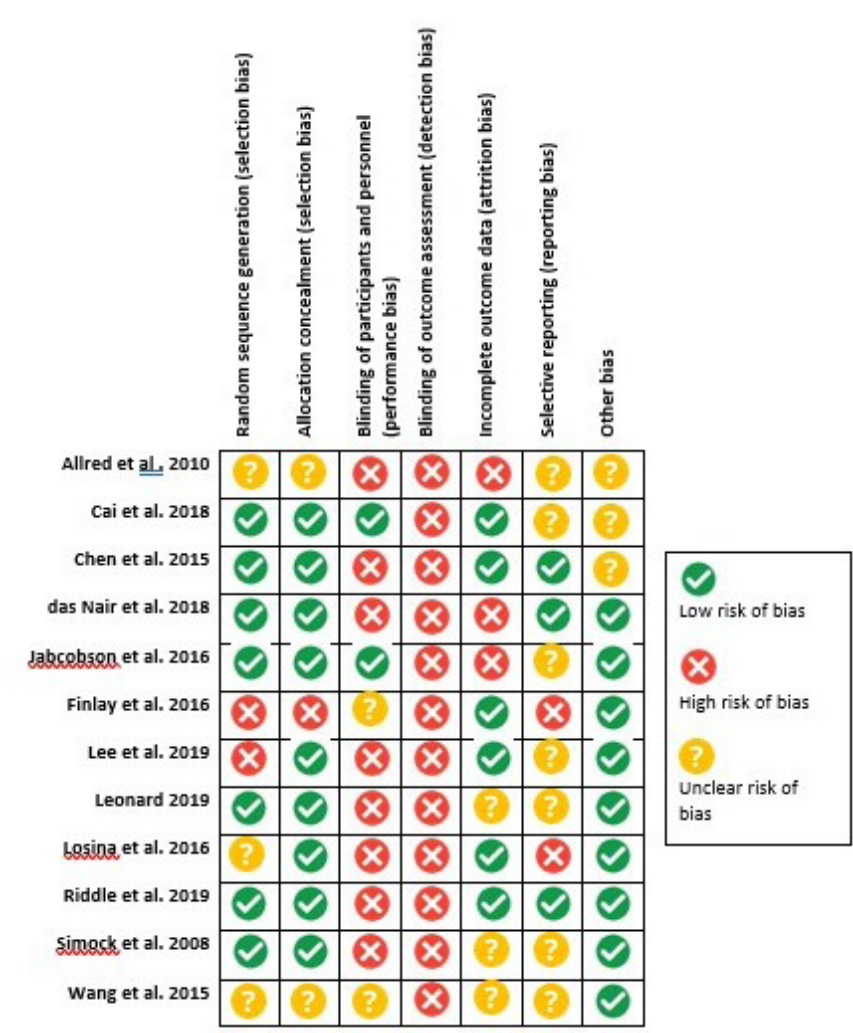

Figure 2 Risk-of-bias summary table (randomised controlled trials).

study was classified as combined relaxation/mindfulness and cognitive; this included a pain coping skills programme. The pain coping skills programme was a complex intervention including multimodal components.

\section{Music}

Five single-centre RCTs with 256 participants evaluated the effectiveness of music therapy for reducing acute postoperative pain during the inpatient stay after surgery. ${ }^{37} 384247$ All studies had high or unclear risk of bias for two or more domains, with blinding of participants and personnel being a high or unclear risk of bias for all studies. Four studies had high or unclear risk of bias for selective reporting. A 2-arm RCT with 75 participants compared listening to a compact disc of 'easy listening' music on headphones for $20 \mathrm{~min}$ before and after first postsurgical ambulation to a 20 min quiet rest period found no differences in mean VAS pain score between groups at any timepoint. ${ }^{38}$ A 2-arm RCT with 30 participants which compared listening to soothing piano and Chinese violin music through broadcast speakers for $30 \mathrm{~min}$ in the preoperative ward, $30 \mathrm{~min}$ in the surgical room waiting area, and 1 hour in postoperative recovery to usual care found no differences in VAS pain score between groups on the ward after surgery. ${ }^{40}$ A 5 -arm RCT with 89 participants which compared listening to 12-15 min of instrument only music with varying degrees of harmonicity and rhythmicity on headphones once per day for 3 days post surgery to wearing headphones with no input found no differences in NRS or VRS pain scores between groups on postoperative days $1-3 .^{42} \mathrm{~A}$ 2-arm RCT with 30 participants which compared patientselected music played on headphones during surgery to white noise found the intervention group reported lower mean VAS pain scores at 3 hours (1.5 (SD 1.4) vs 3.9 (SD $3.4) ; \mathrm{p}=0.01$ ) and 24 hours (2.4 (SD 1.7) vs 4.1 (SD 2.9); $\mathrm{p}=0.04$ ) post surgery. ${ }^{37}$ A 2-arm RCT with 32 patients compared music therapist-delivered live music during a 5 min physiotherapy pedalling exercise to no music found no mean difference in NRS pain scores between groups at the 2 min break timepoint and at the 4 min endpoint after the pedalling exercise.

\section{Guided imagery and music}

One single-centre RCT evaluated the effectiveness of guided imagery on outcomes post surgery. ${ }^{43}$ The study was at high or unclear risk of bias for three domains. The 2-arm RCT with 82 participants was multimodal and compared 19-21 min of audio-recorded guided imagery with the addition of soothing instrumental background music listened to every day for 2 weeks before surgery and 3 weeks after surgery to a control group who received 17-21 min of commercially available spoken word audio recordings (eg, poetry, short stories, essays) at the same timepoints. Pain was assessed preoperatively, on the day of surgery, 3 weeks post surgery, and 6 months post surgery using the WOMAC pain score and VAS pain score. No comparisons were made between trial arms.

\section{Hypnosis}

One single-centre RCT evaluated the effectiveness of a prerecorded hypnotic audio recording on outcome post surgery. ${ }^{48}$ The study was high or unclear risk of bias for four domains. The 3-arm RCT with 24 patients compared 35 min prerecorded hypnosis audio listened to at least once presurgery and at least once 24 hours post surgery to minimal treatment effect (psychoeducation, diaphragmatic breathing, relaxing music), and treatment as usual. Pain was assessed using a pain NRS preoperatively, daily until discharge, and then at 1 month, 3 months, and 6 months. Differences in mean pain ratings between the groups were small at 72 hours (1.77 vs 2.23 vs 2.59$)$ and 6 months (1.4 vs 1.73 s 2.23 ).

\section{Progressive muscle relaxation with biofeedback}

One single-centre RCT evaluated the effectiveness of progressive muscle relaxation with biofeedback on pain during continuous passive motion therapy. ${ }^{46}$ The study had unclear risk of bias for five domains and high risk of bias for blinding of outcome assessment. The 2-arm RCT with 66 participants was multimodal and compared $30 \mathrm{~min}$ of training on biofeedback-assisted progressive muscle relaxation skills on the day before surgery and during $30 \mathrm{~min}$ sessions of continuous passive motion therapy two times a day for 5 days post surgery to standard continuous passive motion therapy. Pain was assessed on days 1-5 after surgery before and after continuous passive motion therapy using an NRS pain score. The intervention 
showed a significant between group effect $(\mathrm{p}<0.001)$ with the intervention group reporting lower NRS pain scores compared with the control group on all 5 days.

\section{Pain coping skills programme}

One 5-centre RCT evaluated the effectives of a pain coping skills training programme for patients who catastrophise about pain before TKR. ${ }^{49}$ The study was at high risk of bias for two domains, blinding participants and personnel and blinding of outcome assessment. The 3-arm RCT with 402 patients compared an eight-session pain coping skills programme to arthritis education and to usual care. The pain coping skills programme comprised eight 50 min sessions over a 2-month period beginning 2 weeks before surgery and ending 6 weeks after surgery. One session was in person with remaining sessions via telephone. The programme was a complex multimodal intervention and included sessions on cognitive restructuring, thought identification and challenging, self-calming and relaxation techniques, and activity management. Arthritis education follows the same schedule, although without the psychological components. Pain was assessed by the WOMAC Pain Scale at baseline and 2, 6, and 12 months post surgery. No differences were found in mean WOMAC pain treatment scores or group-by-time interaction.

\section{Enhanced postoperative recovery using motivational interviewing}

One single-centre RCT evaluated the effectiveness of an enhanced postoperative recovery programme to improve postoperative functional status. ${ }^{44}$ The study was at high risk of bias for blinding of participants and personnel, blinding of outcome assessment and selective reporting. In this 2-arm RCT with 308 participants, the intervention was an enhanced postoperative recovery programme comprising 10 telephone calls with a navigator over a 6-month postoperative period aimed at identifying postsurgical objectives and improving self-efficacy using motivational interviewing. The control group received usual care including inpatient physiotherapy and outpatient physiotherapy after discharge. Pain was assessed at baseline, 3 month post surgery, and 6 months post surgery using the WOMAC pain score. There were no differences between groups in mean WOMAC pain scores at 6 months post operation.

\section{CBT programmes}

Two RCTs (one pilot and one feasibility) with 150 participants evaluated the effectiveness of CBT-based programmes. ${ }^{3941}$ Both studies were at low risk of bias for four domains, with one having unclear risk of bias for selective outcome reporting and other bias, ${ }^{39}$ and one with high risk of bias for blinding of participants and personnel, blinding of outcome assessment, and incomplete outcome data. ${ }^{41}$ One 2-arm pilot RCT with 100 participants evaluated use of CBT that aimed to reduce kinesiophobia (fear of movement) post surgery when compared with standard care. ${ }^{39}$ Four tailored sessions of 30 min each were delivered individually. Between group difference was found with reduction in pain NRS scores of 5.63 (SD 0.73) in the intervention group compared with 6.27 (SD 0.86) in the standard care group demonstrated at 6 -month follow-up $(\mathrm{p}=0.003)$. One 2-arm feasibility RCT with 50 participants compared the use of a CBT-based programme of up to 101 hour sessions for reducing anxiety and depression to standard care. ${ }^{41}$ No between group differences in pain measured using the WOMAC pain score were found at 4-month or 6-month follow-up, and no between group difference in mood was found at 4-month or 12-month follow-up measured using the Beck Anxiety Inventory and Depression Inventory.

\section{Intervention reporting standards}

Table 2 documents the extent to which the included studies adhere to the TIDieR guidelines for reporting on interventions. Overall, all studies provided the name of the intervention, rationale for the intervention, procedures, and how the intervention was delivered. Nine studies provided information about who provided the intervention and their training, with two studies providing partial details and one study providing no details. Ten studies provided details on where the intervention was carried out, and 11 studies reported on the timing and intensity of the intervention with 1 study providing partial details. Reporting of tailoring, modifications and fidelity/ adherence was generally poor: only one study provided information about both tailoring and modifications to the intervention, and only five studies provided information on fidelity/adherence (planned or actual) with one study providing partial details.

Overall, although no studies provided information relating to all TIDieR domains, all studies provided details on at least 7 out of 10 the domains, with most providing details on 8 or more domains.

\section{Ongoing research}

Three published protocols for RCTs were identified in searches that would meet the inclusion criteria of the systematic review and have not yet been published. One study is focused on cognitive/behavioural interventions delivered by physiotherapists for patients with high pain catastrophising before TKR. ${ }^{50}$ One RCT will evaluate a theory-based telephone-delivered patient selfmanagement support intervention to enhance adherence to exercise after TKR. ${ }^{51}$ The final study is focused on a presurgery group-based mindfulness training programme to improve postoperative pain. ${ }^{52}$

\section{DISCUSSION}

This systematic review identified 12 RCTs that have evaluated the effectiveness of psychological interventions for improving pain outcomes after TKR surgery, with the predominant focus on mindfulness and relaxation. The largest group of interventions was music, and the majority of studies evaluated the effectiveness of interventions for reducing acute-postoperative pain. Pooling of data in 


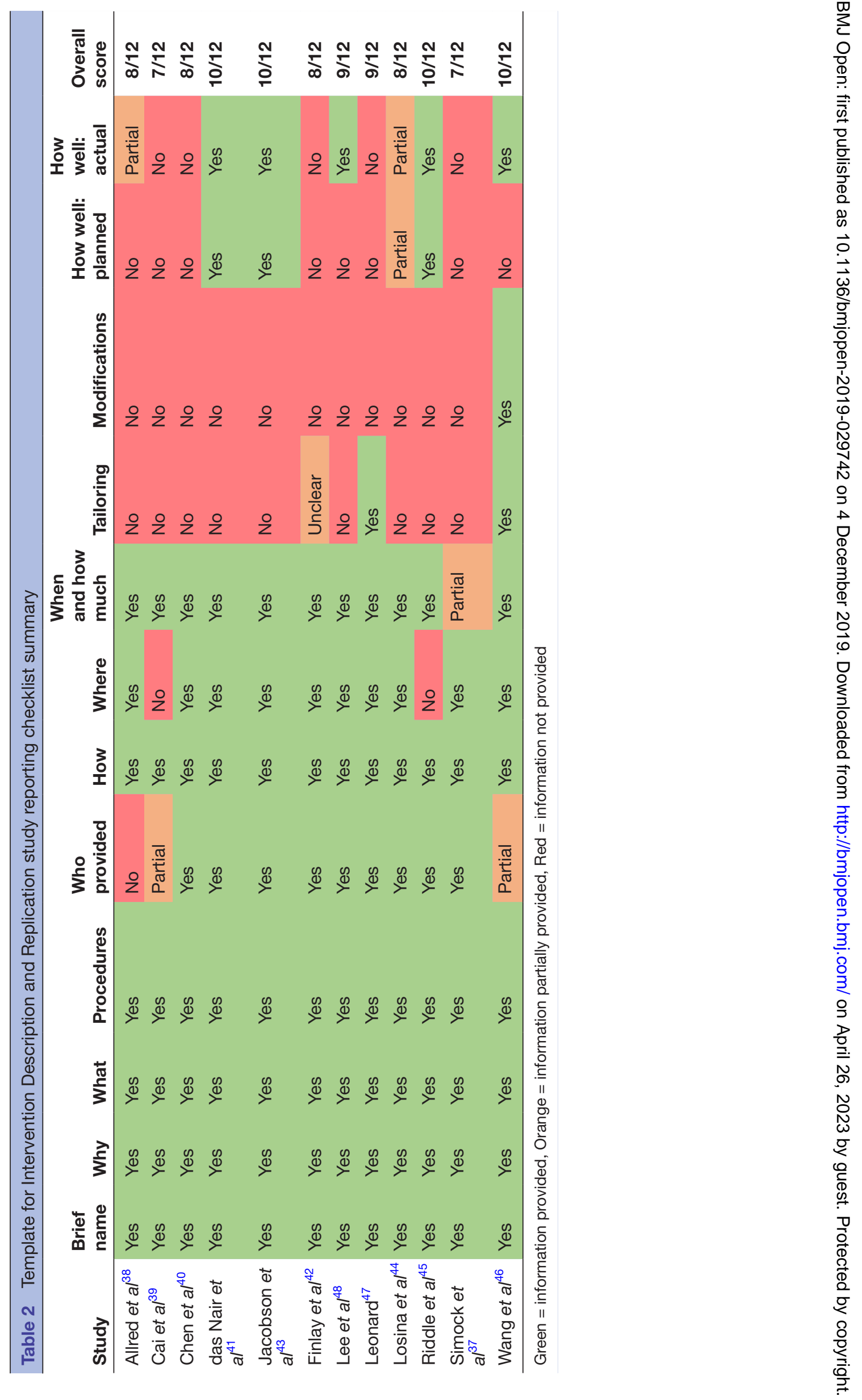


meta-analysis was not possible due to the high heterogeneity between the interventions evaluated. One study did not compare outcomes between the intervention and control group and all RCTs had high or unclear risk of bias for at least three domains. Therefore, it was not possible to make any conclusive statements about the overall effectiveness of psychological interventions for pain outcomes after TKR. However, some promising areas for future research were identified including the use of CBT to reduce kinesiophobia ${ }^{39}$ and the use of progressive muscle relaxation during continuous passive motion therapy. ${ }^{46}$ Use of the TIDieR checklist as a framework highlighted a need for improvements in the reporting of interventions, particularly in relation to fidelity/adherence.

This review included studies evaluating the effectiveness of psychological interventions on both short-term and long-term outcomes for pain. However, the majority of included studies focused on acute postoperative pain. While ensuring optimal management of short-term pain is important, chronic pain is a substantial issue for TKR patients with up to $20 \%$ reporting long-term pain after surgery. ${ }^{7-9}$ Chronic pain after TKR is associated with functional limitations and reduced activity levels and can have a substantial negative impact on well-being and quality of life. ${ }^{53-57}$ Treatment and investigations for patients who have ongoing pain after TKR come at a cost to the NHS that is above costs for those for whom there is no ongoing pain. ${ }^{11}$ All the studies in this review delivered interventions preoperatively, during the immediate postoperative recovery period, or both. We were unable to discern if psychological interventions are more or less effective dependent on the timing of delivery. In addition, pain outcomes and mechanisms may differ between acute postoperative pain due to surgical recovery and chronic postsurgical pain, requiring different intervention approaches. Further robust research is needed to evaluate psychological interventions aimed at targeting longterm or chronic pain after TKR, in addition to during the immediate postoperative recovery period.

Only one study in this review provided data on a harm outcome. The assessment of harm outcomes, such as serious adverse events, within interventions is vital for patient safety. However, unlike in trials of pharmacological treatments where monitoring and reporting of adverse events are mandatory, psychological interventions are rarely subject to the same scrutiny. ${ }^{58}$ There is increasing recognition that harm may arise from psychological interventions and that these outcomes should be considered both at the development stage, as seen in dark logic models, ${ }^{59}$ and at the intervention reporting stage. ${ }^{60}$ In 2004, the Consolidated Standards of Reporting Trials group suggested 10 new recommendations for harm reporting in RCTs including explanations and examples of proper reporting. ${ }^{61}$ Despite all RCTs included in this review being published after these recommendations, only one included harms data. This demonstrates a need for improvement in reporting of harms related to psychological interventions.
The purpose of this systematic review was to evaluate the effectiveness of psychological interventions specifically for improving pain outcomes after TKR. Many of the interventions included in the review have been the focus of broader, intervention-specific systematic reviews. For example, a systematic review and meta-analysis of perioperative music interventions found that they reduced postoperative pain in surgical patients. ${ }^{62}$ Interventions using cognitive behavioural modalities have been found to have small benefits for older adults with chronic pain. ${ }^{63}$ Therefore, the wider literature suggests that some psychological interventions are effective at reducing pain severity in mixed patient populations. However, our review highlighted the relative paucity of robust interventions focused on patients undergoing TKR.

The current evidence base is primarily focused on music interventions. Only a small number of trials evaluating interventions have been based on psychological theory or including recognised approaches to psychological and behavioural change, ${ }^{64}$ such as CBT and acceptancebased therapies, or interventions targeting particular psychological traits, such as anxiety, depression, or pain catastrophising, which are all linked to pain. ${ }^{22-25}$ This makes it challenging to identify the 'active ingredients' of the interventions, or by which mechanisms these interventions may be able to effect change. However, more recent studies based on CBT to address specific risk factors such as kinesiophobia and anxiety and depression are now emerging and demonstrate promising results. This indicates that a more targeted and individually tailored approach to psychological interventions may be of greater benefit to the patient population. In addition, there are ongoing trials of psychological interventions, for example, interventions that address catastrophising, ${ }^{50}$ which will add to the evidence base.

\section{Evaluation of reporting standards}

Reporting standards for all included studies were assessed using the TIDieR checklist, with 10 out of the 12 studies published after these guidelines had become available. Reporting completeness was high for intervention's rationale, content, and procedure. However, 10 out of 12 studies did not include any information on tailoring or modifications, and 7 out of 12 did not include adequate information on fidelity and adherence. While tailoring and modification may not have been relevant to many of the standardised interventions evaluated, fidelity and adherence are crucial for accurate interpretation of treatment effects. Psychological interventions are often complex and may involve multiple intervention components, dose intensities, and dose durations. In addition, many psychological interventions are designed to use an individualised approach that accommodates particular individual needs and contexts. ${ }^{6566}$ Due to this complexity, accurate reporting of the implementation and adherence of psychological interventions is vital in order to understand fully the intervention's effects and to inform practice. While the TIDieR guidelines provide a clear 
checklist for minimum information inclusion, including fidelity, they do not provide guidance on how to assess fidelity. To address this issue, additional guidelines have been published to guide fidelity reporting and improve transparency; ${ }^{67} 68$ however, these results of this review demonstrate that there are ongoing issues with the implementation of these guidelines.

\section{Strengths and limitations}

This systematic review has strengths and limitations which should be considered when interpreting the findings. The review was conducted following Cochrane guidance to ensure the methodology was robust and systematic. ${ }^{31}$ RCTs were eligible for inclusion, and published protocols were identified to highlight ongoing research that is likely to add to the existing evidence base. Opportunities for pooling of data in meta-analysis were limited because of heterogeneity in the content, duration, and intensity of the interventions, and conclusions are therefore based on narrative synthesis. Psychological interventions are often complex in nature and may contain multimodal components. To further explore this complexity, tools to aid in the disaggregation of intervention components and categorise levels of intervention complexity, such as iCAT (an intervention complexity assessment tool for systematic reviews), would be of benefit in future reviews. Secondary outcomes were poorly reported across studies with high heterogeneity in the measures used. The primary harm outcome of serious adverse events was inconsistently and poorly reported with only one trial including details on serious adverse events but no a priori definition, an issue which is common in both trials of pain interventions ${ }^{69} 70$ and psychological interventions, ${ }^{5871}$ and therefore the safety of these interventions could not be evaluated. The unclear or high risk-of-bias ratings for many domains of the included studies highlights the need for more rigorous methodological conduct and reporting in studies on this topic. However, despite these limitations, this review provides a comprehensive overview of studies evaluating the effectiveness of psychological interventions for improving pain outcomes after TKR, and the findings have a number of methodological implications for future studies.

\section{CONCLUSION}

This review highlights the need for more evidence about psychological interventions for improving pain outcomes after TKR. Given the high prevalence of acute and chronic pain after TKR, it is important that interventions that may improve pain outcomes are evaluated in high-quality RCTs. This review also highlights substantial ongoing issues in the reporting of interventions, particularly in relation to harm outcomes and intervention fidelity. Guidelines for the reporting of both harm and fidelity do exist and future interventions should implement these guidelines in order to improve reporting standards. Due to the pervasiveness of these problems, research in this area would also benefit from work exploring barriers to guideline implementation. Psychological interventions are broad, encompassing a wide variety of approaches with varying degrees of complexity and specificity. Future development of psychological interventions for people undergoing TKR would benefit from foundation on clearly identified psychological theory, behaviour change mechanisms, or targeting specific psychological traits linked with poor outcomes after TKR, such as anxiety, depression, and pain catastrophising.

\section{Author affiliations}

${ }^{1}$ National Institute for Health Research Bristol Biomedical Research Centre, University Hospitals Bristol NHS Foundation Trust and University of Bristol, Bristol, United Kingdom

${ }^{2}$ Musculoskeletal Research Unit, Translational Health Sciences, Bristol Medical School, University of Bristol, Bristol, UK

${ }^{3}$ Division of Primary Care, School of Medicine, University of Nottingham, Nottingham, UK

${ }^{4}$ Pain Centre Versus Arthritis, City Hospital, University of Nottingham, Nottingham, United Kingdom

\section{Twitter Katie Whale @whalekatie}

Contributors KW: lead author, protocol writing, data searches, data screening, data extraction, manuscript writing and preparation VW: protocol writing, data screening, data extraction and manuscript writing. AB: protocol writing, data searches, methodological design and manuscript review. JR: data extraction and manuscript review. KV: study inclusion review and manuscript review. RG-H: protocol writing and manuscript review.

Funding This study was supported by the NIHR Biomedical Research Centre at University Hospitals Bristol NHS Foundation Trust and the University of Bristol, and the NIHR Nottingham Biomedical Research Centre, Nottingham University Hospitals NHS Trust.

Disclaimer The views expressed in this publication are those of the author(s) and not necessarily those of the NHS, the National Institute for Health Research or the Department of Health.

Competing interests None declared.

Patient consent for publication Not required.

Provenance and peer review Not commissioned; externally peer reviewed.

Data availability statement All data relevant to the study are included in the article or uploaded as supplementary information.

Open access This is an open access article distributed in accordance with the Creative Commons Attribution Non Commercial (CC BY-NC 4.0) license, which permits others to distribute, remix, adapt, build upon this work non-commercially, and license their derivative works on different terms, provided the original work is properly cited, appropriate credit is given, any changes made indicated, and the use is non-commercial. See: http://creativecommons.org/licenses/by-nc/4.0/.

\section{REFERENCES}

1 HQIP. National joint registry 15th annual report; 2018.

2 Project SA. Annual report 2018 NHS National Services Scotland; 2018.

3 Anderson JG, Wixson RL, Tsai D, et al. Functional outcome and patient satisfaction in total knee patients over the age of $75 . J$ Arthroplasty 1996;11:831-40.

4 Heck DA, Robinson LS, Patridge CM, et al. Patient outcomes after knee replacement. Clin Orthop Relat Res 1998;358:93-110.

5 Robertsson O, Dunbar M, Pehrsson T, et al. Patient satisfaction after knee arthroplasty: a report on 27,372 knees operated on between 1981 and 1995 in Sweden. Acta Orthop Scand 2000;71:262-7.

6 Wylde V, Rooker J, Halliday L, et al. Acute postoperative pain at rest after hip and knee arthroplasty: severity, sensory qualities and impact on sleep. Orthop Traumatol Surg Res 2011;97:139-44.

7 Baker PN, van der Meulen JH, Lewsey J, et al. The role of pain and function in determining patient satisfaction after total knee replacement. J Bone Joint Surg Br 2007;89-B:893-900. 
8 Gandhi R, Davey JR, Mahomed NN. Predicting patient dissatisfaction following joint replacement surgery. J Rheumatol 2008;35:2415-8.

9 Kim TK, Chang CB, Kang YG, et al. Causes and predictors of patient's dissatisfaction after uncomplicated total knee arthroplasty. $J$ Arthroplasty 2009;24:263-71.

10 Wylde V, Beswick A, Bruce J, et al. Chronic pain after total knee arthroplasty. EFORT Open Rev 2018;3:461-70.

11 Kassam AM, Dieppe P, Toms AD. An analysis of time and money spent on investigating painful total knee replacements. British Journal of Medical Practitioners 2012;5.

12 Petersen KK, Simonsen O, Laursen MB, et al. Chronic postoperative pain after primary and revision total knee arthroplasty. Clin J Pain 2015;31:1-6.

13 Katz J, Seltzer Ze'ev, Seltzer Z. Transition from acute to chronic postsurgical pain: risk factors and protective factors. Expert Rev Neurother 2009:9:723-44.

14 Lindberg MF, Miaskowski C, RustøEn T, et al. Factors that can predict pain with walking, 12 months after total knee arthroplasty. Acta Orthop 2016;87:600-6.

15 Pinto PR, Mclntyre T, Ferrero R, et al. Risk factors for moderate and severe persistent pain in patients undergoing total knee and hip arthroplasty: a prospective predictive study. PLoS One 2013;8:e73917.

16 Vissers MM, Bussmann JB, Verhaar JAN, et al. Psychological factors affecting the outcome of total hip and knee arthroplasty: a systematic review. Semin Arthritis Rheum 2012;41:576-88.

17 Flor H, Fydrich T, Turk DC. Efficacy of multidisciplinary pain treatment centers: a meta-analytic review. Pain 1992;49:221-30.

18 McCracken LM, Turk DC. Behavioral and cognitive-behaviora treatment for chronic pain: outcome, predictors of outcome, and treatment process. Spine 2002;27:2564-73.

19 Kamper SJ, Apeldoorn AT, Chiarotto A, et al. Multidisciplinary biopsychosocial rehabilitation for chronic low back pain: cochrane systematic review and meta-analysis. BMJ 2015;350:h444.

20 Choi S, O'Hare T, Gollish J, et al. Optimizing pain and rehabilitation after knee arthroplasty: a two-center, randomized trial. Anesth Analg 2016;123:1316-24.

21 Artz N, Elvers KT, Lowe CM, et al. Effectiveness of physiotherapy exercise following total knee replacement: systematic review and meta-analysis. BMC Musculoskelet Disord 2015;16:15.

22 Lewis GN, Rice DA, McNair PJ, et al. Predictors of persistent pain after total knee arthroplasty: a systematic review and meta-analysis. Br J Anaesth 2015;114:551-61.

23 Baert IAC, Lluch E, Mulder T, et al. Does pre-surgical central modulation of pain influence outcome after total knee replacement? A systematic review. Osteoarthritis Cartilage 2016;24:213-23

24 Lopez-Olivo MA, Landon GC, Siff SJ, et al. Psychosocial determinants of outcomes in knee replacement. Ann Rheum Dis 2011;70:1775-81.

25 Shim J, Mclernon DJ, Hamilton D, et al. Development of a clinical risk score for pain and function following total knee arthroplasty: results from the trio study. Rheumatol Adv Pract 2018;2.

26 Nelson EA, Dowsey MM, Knowles SR, et al. Systematic review of the efficacy of pre-surgical Mind-body based therapies on post-operative outcome measures. Complement Ther Med 2013;21:697-711.

27 Bay S, Kuster L, McLean N, et al. A systematic review of psychological interventions in total hip and knee arthroplasty. BMC Musculoskelet Disord 2018;19:201.

28 Sayers A, Wylde V, Lenguerrand E, et al. Rest pain and movementevoked pain as unique constructs in hip and knee replacements. Arthritis Care Res 2016;68:237-45.

29 Glasziou P, Meats E, Heneghan C, et al. What is missing from descriptions of treatment in trials and reviews? $B M J$ 2008;336:1472-4.

30 Hoffmann TC, Glasziou PP, Boutron I, et al. Better reporting of interventions: template for intervention description and replication (TIDieR) checklist and guide. BMJ 2014;348:g1687.

31 Higgins J.P.T, Green S. Cochrane Handbook for Systematic Review of Interventions. Version 5.1.0 The Cochrane Collaboration. 2011.

32 Moher D, Liberati A, Tetzlaff J, et al. Preferred reporting items for systematic reviews and meta-analyses: the PRISMA statement. BMJ 2009;339:b2535.

33 Farrand P, Matthews J, Dickens C, et al. Psychological interventions to improve psychological well-being in people with dementia or mild cognitive impairment: systematic review and meta-analysis protocol. BMJ Open 2016;6:e009713.

34 Higgins JPT, Altman DG, Gøtzsche PC, et al. The Cochrane collaboration's tool for assessing risk of bias in randomised trials. BMJ 2011;343:d5928.
35 Baldwin AL, Vitale A, Brownell E, et al. Effects of Reiki on pain, anxiety, and blood pressure in patients undergoing knee replacement: a pilot study. Holist Nurs Pract 2017;31:80-9.

36 Notte BB, Fazzini C, Mooney RA. Reiki's effect on patients with total knee arthroplasty: a pilot study. Nursing 2016;46:17-23.

37 Simcock XC, Yoon RS, Chalmers P, et al. Intraoperative music reduces perceived pain after total knee arthroplasty: a blinded, prospective, randomized, placebo-controlled clinical trial. J Knee Surg 2008;21:275-8.

38 Allred KD, Byers JF, Sole ML. The effect of music on postoperative pain and anxiety. Pain Manag Nurs 2010;11:15-25.

39 Cai L, Gao H, Xu H, et al. Does a Program Based on Cognitive Behavioral Therapy Affect Kinesiophobia in Patients Following Tota Knee Arthroplasty? A Randomized, Controlled Trial With a 6-Month Follow-Up. J Arthroplasty 2018;33:704-10.

40 Chen H-J, Chen T-Y, Huang C-Y, et al. Effects of music on psychophysiological responses and opioid dosage in patients undergoing total knee replacement surgery. Jpn J Nurs Sci 2015;12:309-19.

41 das Nair R, Mhizha-Murira JR, Anderson P, et al. Home-Based pre-surgical psychological intervention for knee osteoarthritis (HAPPiKNEES): a feasibility randomized controlled trial. Clin Rehabil 2018;32:777-89.

42 Finlay KA, Wilson JA, Gaston P, et al. Post-Operative pain management through audio-analgesia: investigating musical constructs. Psychol Music 2016;44:493-513.

43 Jacobson AF, Umberger WA, Palmieri PA, et al. Guided imagery for total knee replacement: a randomized, placebo-controlled pilot study. J Altern Complement Med 2016;22:563-75.

44 Losina E, Collins JE, Wright J, et al. Postoperative care navigation for total knee arthroplasty patients: a randomized controlled trial. Arthritis Care Res 2016;68:1252-9.

45 Riddle DL, Keefe FJ, Nay WT, et al. Pain coping skills training for patients with elevated pain catastrophizing who are scheduled for knee arthroplasty: a quasi-experimental study. Arch Phys Med Rehabil 2011;92:859-65.

46 Wang T-J, Chang C-F, Lou M-F, et al. Biofeedback relaxation for pain associated with continuous passive motion in Taiwanese patients after total knee arthroplasty. Res Nurs Health 2015;38:39-50.

47 Leonard $\mathrm{H}$. Live music therapy during rehabilitation after total knee arthroplasty: a randomized controlled trial. J Music Ther 2019;56:61-89.

48 Lee JK, Zubaidah JO, Fadhilah ISI, et al. Prerecorded Hypnotic PeriSurgical Intervention to Alleviate Risk of Chronic Postsurgical Pain in Total Knee Replacement: A Randomized Controlled Pilot Study. Int $J$ Clin Exp Hypn 2019;67:217-45.

49 Riddle DL, Keefe FJ, Ang DC, et al. Pain coping skills training for patients who Catastrophize about pain prior to knee arthroplasty: a multisite randomized clinical trial. J Bone Joint Surg Am 2019;101:218-27.

50 Birch S, Stilling M, Mechlenburg I, et al. Effectiveness of a physiotherapist delivered cognitive-behavioral patient education for patients who undergoes operation for total knee arthroplasty: a protocol of a randomized controlled trial. BMC Musculoskelet Disord 2017; $18: 116$

51 Rosal MC, Ayers D, Li W, et al. A randomized clinical trial of a peri-operative behavioral intervention to improve physical activity adherence and functional outcomes following total knee replacement. BMC Musculoskelet Disord 2011;12:226.

52 Dowsey MM, Castle DJ, Knowles SR, et al. The effect of mindfulness training prior to total joint arthroplasty on post-operative pain and physical function: study protocol for a randomised controlled trial. Trials 2014;15:208

53 Breivik H, Collett B, Ventafridda V, et al. Survey of chronic pain in Europe: prevalence, impact on daily life, and treatment. Eur J Pain 2006;10:287-333.

54 Scott KM, Bruffaerts R, Tsang A, et al. Depression-anxiety relationships with chronic physical conditions: results from the world mental health surveys. J Affect Disord 2007;103:113-20.

55 Closs SJ, Staples V, Reid I, et al. The impact of neuropathic pain on relationships. J Adv Nurs 2009;65:402-11.

56 Núñez M, Núñez E, del Val JL, Luis del Val J, et al. Health-Related quality of life in patients with osteoarthritis after total knee replacement: factors influencing outcomes at 36 months of followup. Osteoarthritis Cartilage 2007:15:1001-7.

57 Bourne RB, Chesworth BM, Davis AM, et al. Patient satisfaction after total knee arthroplasty: who is satisfied and who is not? Clin Orthop Relat Res 2010;468:57-63.

58 Grant S, Montgomery P, Hopewell S, et al. Developing a reporting guideline for social and psychological intervention trials. Res Soc Work Pract 2013;23:595-602. 
59 Bonell C, Jamal F, Melendez-Torres GJ, et al. 'Dark logic': theorising the harmful consequences of public health interventions. J Epidemiol Community Health 2015;69:95-8.

60 Duggan C, Parry G, McMurran M, et al. The recording of adverse events from psychological treatments in clinical trials: evidence from a review of NIHR-funded trials. Trials 2014;15:335.

61 loannidis JPA, Evans SJW, Gøtzsche PC, et al. Better reporting of harms in randomized trials: an extension of the CONSORT statement. Ann Intern Med 2004;141:781-8.

62 Kühlmann AYR, de Rooij A, Kroese LF, et al. Meta-Analysis evaluating music interventions for anxiety and pain in surgery. Br J Surg 2018;105:773-83.

63 Niknejad B, Bolier R, Henderson CR, et al. Association between psychological interventions and chronic pain outcomes in older adults: a systematic review and meta-analysis. JAMA Intern Med 2018;178:830-9.

64 Michie S, Richardson M, Johnston M, et al. The behavior change technique taxonomy (V1) of 93 hierarchically clustered techniques: building an international consensus for the reporting of behavior change interventions. Ann Behav Med 2013;46:81-95.
65 Bonell C, Fletcher A, Morton M, et al. Realist randomised controlled trials: a new approach to evaluating complex public health interventions. Soc Sci Med 2012;75:2299-306.

66 Hawe P, Shiell A, Riley T. Complex interventions: how "out of control" can a randomised controlled trial be? BMJ 2004;328:1561-3.

67 Gearing RE, El-Bassel N, Ghesquiere A, et al. Major ingredients of fidelity: a review and scientific guide to improving quality of intervention research implementation. Clin Psychol Rev 2011;31:79-88.

68 Mayo-Wilson E, Montgomery P, Hopewell S, et al. Developing a reporting guideline for social and psychological intervention trials. $\mathrm{Br}$ J Psychiatry 2013;203:250-4.

69 Alviar MJM, Hale T, Dungca M, et al. Pharmacologic interventions for treating phantom limb pain. Cochrane Database Syst Rev 2016;10.

70 Wylde V, Dennis J, Beswick AD, et al. Systematic review of management of chronic pain after surgery. Br J Surg 2017;104:1293-306.

71 Chambless DL, Ollendick TH. Empirically supported psychological interventions: controversies and evidence. Annu Rev Psychol 2001;52:685-716. 